$12-1-2001$

\title{
Semiclassical Scattering of an Electric Dipole Source Inside a Spherical Particle
}

James A. Lock

Cleveland State University, j.lock@csuohio.edu

Follow this and additional works at: https://engagedscholarship.csuohio.edu/sciphysics_facpub

Part of the Physics Commons

How does access to this work benefit you? Let us know!

\section{Publisher's Statement}

This paper was published in Journal of the Optical Society of America A: Optics Image Science and Vision and is made available as an electronic reprint with the permission of OSA. The paper can be found at the following URL on the OSA website: http://www.opticsinfobase.org/josaa/ abstract.cfm?URI=josaa-18-12-3085. Systematic or multiple reproduction or distribution to multiple locations via electronic or other means is prohibited and is subject to penalties under law.

\section{Original Citation}

Lock, James A. "Semiclassical Scattering of an Electric Dipole Source Inside a Spherical Particle." Journal of the Optical Society of America A: Optics Image Science and Vision 18 (2001): 3085-3097.

\section{Repository Citation}

Lock, James A., "Semiclassical Scattering of an Electric Dipole Source Inside a Spherical Particle" (2001). Physics Faculty Publications. 29.

https://engagedscholarship.csuohio.edu/sciphysics_facpub/29

This Article is brought to you for free and open access by the Physics Department at EngagedScholarship@CSU. It has been accepted for inclusion in Physics Faculty Publications by an authorized administrator of EngagedScholarship@CSU. For more information, please contact library.es@csuohio.edu. 


\title{
Semiclassical scattering of an electric dipole source inside a spherical particle
}

\author{
James A. Lock \\ Department of Physics, Cleveland State University, Cleveland, Ohio 44115
}

Received March 12, 2001; accepted May 22, 2001; revised manuscript received June 8, 2001

\begin{abstract}
Semiclassical scattering phenomena appearing in the far-zone scattered intensity of a point source of electromagnetic radiation inside a spherical particle are examined in the context of both ray theory and wave theory, and the evolution of the phenomena is studied as a function of source position. A number of semiclassical effects that do not occur for plane-wave scattering by the sphere appear prominently for scattering by an interior source. These include a series of scattering resonances and a new family of rainbows in regions of otherwise total internal reflection. Diffractive effects accompanying the semiclassical phenomena are also examined. (C) 2001 Optical Society of America
\end{abstract}

OCIS codes: $290.0290,290.4020,290.5850,260.2510$.

\section{INTRODUCTION}

Although Mie theory provides the exact solution to the electromagnetic boundary-value problem of a plane wave scattered by a spherical particle, the solution takes the form of an infinite series of partial-wave contributions ${ }^{1-3}$ that converges very slowly in the semiclassical limit, where the particle radius is much larger than the wavelength of the incident light. As a result, identification of the portion of the Mie partial-wave sum responsible for the various phenomena of semiclassical scattering such as rainbows and glories is not straightforward and requires the Debye series expansion of the partial wave scattering amplitudes, ${ }^{4,5}$ the stationary phase arguments, ${ }^{6}$ and the complex angular momentum formalism. ${ }^{7-9}$

The exact solution to the related electromagnetic boundary-value problem of scattering by an arbitrarily positioned, arbitrarily oriented electric dipole source inside a spherical particle has also been derived. ${ }^{10-16}$ This situation describes, for example, a liquid droplet containing molecules that fluoresce. Although scattering in the short-wavelength limit by a sphere containing an interior source has been computed by using both wave theory ${ }^{17}$ and ray theory, ${ }^{18-22}$ a detailed physical interpretation of the angular dependence of the scattered intensity has not been given. Thus, to provide insight into the scattering process, this paper catalogs the semiclassical phenomena appearing in the scattered intensity of an interior source by using both ray theory and wave theory methods.

We find that all the semiclassical phenomena occurring in Mie scattering of a plane wave by a spherical particle, e.g., rainbows, glories, axial focusing, ${ }^{23}$ and rainbowenhanced glories, ${ }^{24}$ also occur for scattering by an interior point source. But a number of additional phenomena not found in Mie scattering occur as well. In particular, the scattered intensity in ray theory vanishes in a number of angular regions as a result of total internal reflection. Two rainbows, rather than only one, occur for each ray family making a given number of reflections inside the sphere before exiting. The additional rainbow for each ray family, including the zero-order rainbow for transmitted rays, ${ }^{25,26}$ occurs within the family's total internal reflection region. Thus we call these total internal reflection rainbows. Also, as the source moves inward toward the center of the sphere, each total internal reflection rainbow evolves into a scattering resonance.

The body of this paper is organized as follows. In Section 2, we examine total internal reflection regions, axial focusing, rainbows, glories, resonances, and total internal reflection rainbows for scattering by an interior isotropic point source in the context of ray theory. We also consider the specific example of a point source in a water droplet to illustrate the variety of different ways in which semiclassical phenomena manifest themselves in the scattered intensity. In Section 3, we examine scattering of an interior electric dipole source in wave theory and derive the Debye series expansion of the partial wave scattering amplitudes. We again consider the specific example of an electric dipole source in a water droplet for two situations where scattering by the dipole source is quantitatively described by isotropic source scattering in ray theory. Last, in Section 4, we inquire whether any of the semiclassical phenomena remain visible in the scattered intensity when a liquid droplet containing a weak uniform concentration of fluorescing molecules is illuminated by a plane wave.

\section{RAY SCATTERING OF AN ISOTROPIC INTERIOR POINT SOURCE}

\section{A. Geometry and Ray Families}

Consider a monochromatic isotropic point source of power $P$ and free-space wavelength $\lambda$ located inside a sphere of radius $a$ and refractive index $N>1$ in the shortwavelength limit, where $a \gg \lambda$. We employ the coordinate system in which the source is located at $z_{0}$ on the positive $z$ axis. Since the source is assumed to be isotropic, the scattered intensity depends only on the spherical coordinate system scattering angle $\theta$ and is independent 
of the angle $\phi$ in the $x y$ plane. Let $\alpha$ be the angle that a ray emitted by the source makes with the positive $z$ axis, with $0 \leqslant \alpha \leqslant \pi$, as is shown in Fig. 1 . The angle of incidence of the ray at the sphere surface, $\theta_{i}$, and the refracted angle of the exiting ray, $\theta_{t}$, are related to $\alpha$ by

$$
\begin{aligned}
& \sin \left(\theta_{i}\right)=q \sin (\alpha), \\
& \sin \left(\theta_{t}\right)=N \sin \left(\theta_{i}\right),
\end{aligned}
$$

with

$$
q \equiv z_{0} / a
$$

and $0 \leqslant q<1$. The Fresnel coefficients for transmission and reflection of the ray at the sphere surface, $T\left(\theta_{i}\right)$ and $R\left(\theta_{i}\right)$, are taken to be

$$
\begin{aligned}
& T\left(\theta_{i}\right)=4 N \cos \left(\theta_{i}\right) \cos \left(\theta_{t}\right) /\left[N \cos \left(\theta_{i}\right)+\cos \left(\theta_{t}\right)\right]^{2}, \\
& R\left(\theta_{i}\right)=\left[N \cos \left(\theta_{i}\right)-\cos \left(\theta_{t}\right)\right]^{2} /\left[N \cos \left(\theta_{i}\right)+\cos \left(\theta_{t}\right)\right]^{2} .
\end{aligned}
$$

Equations (4) are appropriate for scalar waves and the transverse electric polarization of electromagnetic waves.

Consider a ray path containing $p-1$ internal reflections before exiting the sphere, with $p \geqslant 1$. The deflection angle $\Theta$ of the scattered ray with respect to the positive $z$ axis is

$$
\Theta=(p-1) \pi+\alpha-(2 p-1) \theta_{i}+\theta_{t} .
$$

The scattering angle $\theta$ of the ray is constrained to the interval $0 \leqslant \theta \leqslant \pi$ and is related to $\Theta$ by

$\theta=\left\{\begin{array}{ll}\Theta-2 M \pi & \text { if } 2 M \pi \leqslant \Theta \leqslant(2 M+1) \pi, \\ (2 M+2) \pi-\Theta & \text { if }(2 M+1) \pi \\ & <\Theta<(2 M+2) \pi\end{array}\right.$,

where $M$ is a positive integer. Whereas trajectories of light rays are parameterized most simply by the deflection angle, the scattered intensity is computed in wave theory in terms of the scattering angle.

The scattered intensity of the $p$ family of rays a distance $r$ from the center of the sphere in the scattering far zone is found from flux conservation to be

$$
I(\theta)=P \sin (\alpha)\left[R\left(\theta_{i}\right)\right]^{p-1} T\left(\theta_{i}\right) /\left[4 \pi r^{2} \sin (\theta)|\mathrm{d} \Theta / \mathrm{d} \alpha|\right],
$$

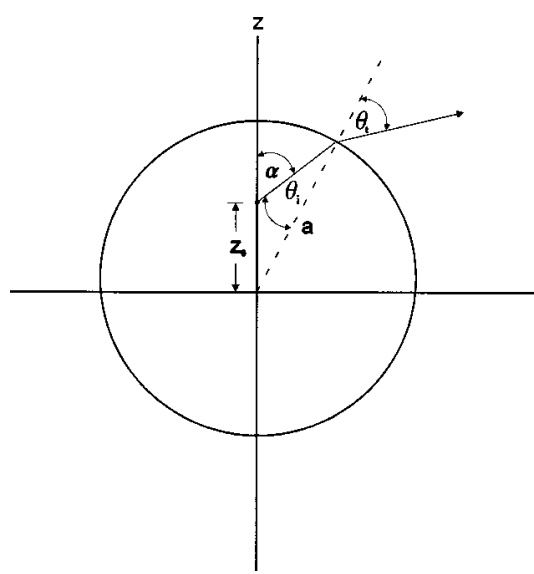

Fig. 1. $p=1$ ray trajectory. The source is located at $z_{0}$ on the $z$ axis of the sphere. The ray makes an angle $\alpha$ with the $z$ axis, and the angles of incidence and transmission at the sphere surface are $\theta_{i}$ and $\theta_{t}$. where

$$
\begin{aligned}
\mathrm{d} \Theta / \mathrm{d} \alpha= & 1-(2 p-1) q \cos (\alpha) / \cos \left(\theta_{i}\right) \\
& +N q \cos (\alpha) / \cos \left(\theta_{t}\right) .
\end{aligned}
$$

The angular structure of Eqs. (7) and (8) is dominated by six semiclassical scattering phenomena. These are regions of total internal reflection (TIR), where the scattered intensity in ray theory vanishes; rainbows $(R)$, glories $(\mathrm{G})$, and axial focusing (AF), where the intensity scattered in a particular direction in ray theory diverges; scattering resonances (RES), where the scattered intensity in a narrow angular interval is large but finite; and total internal reflection rainbows (TIRR), where the scattered intensity diverges in a total internal reflection region. We examine the behavior of each of these phenomena as a function of the source position $q$ for fixed $N$ in Subsections 2.B-2.E.

\section{B. Regions of Total Internal Reflection}

If an interior point source is sufficiently close to the sphere surface, some of the rays emanating from it are incident on the surface past the critical angle for total internal reflection and do not exit the sphere. This is illustrated in Fig. 2 for the $p=1$ ray family. From Eqs. (1) and (2), the critical angle corresponds to $N q \sin (\alpha)=1$. Thus total internal reflection does not occur when the source is near the center of the sphere, i.e., $q<q_{c}$, where

$$
q_{c} \equiv 1 / N \text {. }
$$

For the source position $q=q_{c}$, total internal reflection occurs only for the $\alpha=\pi / 2$ member of the $p$ ray family, corresponding to the deflection angle

$$
\Theta_{\mathrm{TIR}}^{0} \equiv p \pi-(2 p-1) \sin ^{-1}(1 / N) .
$$

For $q>q_{c}$, it occurs for the rays

$$
\alpha_{\mathrm{TIR}}^{-} \leqslant \alpha \leqslant \alpha_{\mathrm{TIR}}^{+},
$$

with

$$
\alpha_{\mathrm{TIR}}^{-}=\sin ^{-1}(1 / N q), \quad \alpha_{\mathrm{TIR}}^{+}=\pi-\sin ^{-1}(1 / N q),
$$

corresponding to the deflection angle interval

$$
\Theta_{\mathrm{TIR}}^{-} \leqslant \Theta \leqslant \Theta_{\mathrm{TIR}}^{+},
$$

with

$$
\Theta_{\mathrm{TIR}}^{ \pm}=\Theta_{\mathrm{TIR}}^{0} \pm\left[\pi / 2-\sin ^{-1}(1 / N q)\right] .
$$

In the vicinity of the critical source position, $q=q_{c}+\epsilon$ with $\epsilon \ll 1$, Eq. (14) reduces to

$$
\Theta_{\mathrm{TIR}}^{ \pm}=\Theta_{\mathrm{TIR}}^{0} \pm(2 N \epsilon)^{1 / 2}+O(\epsilon) .
$$

These regions differ from the total internal reflection of the interior fields of morphology-dependent resonances (MDRs) in a number of ways. First, for an interior source these regions are bounded by the deflection angles $\Theta_{\mathrm{TIR}}^{ \pm}$, while for MDRs total internal reflection occurs over the entire sphere surface. Also, the ray components of the incident plane wave that excite MDR rays inside the sphere have impact parameters that classically miss striking the sphere and, as a result, couple to the interior by means of tunneling. 


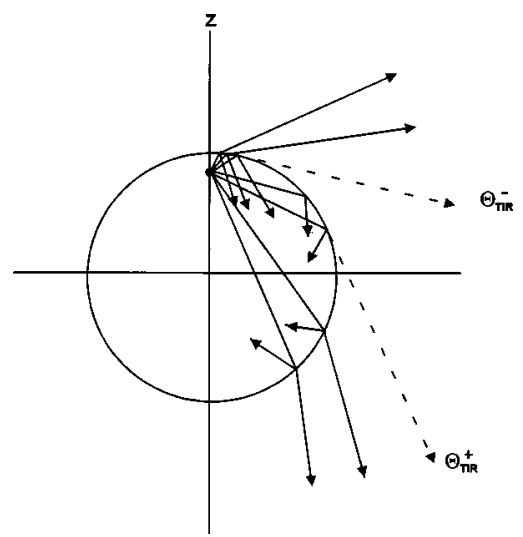

Fig. 2. $p=1$ family of scattered rays. $p=1$ rays do not exit the sphere between $\Theta_{\mathrm{TIR}}^{-}$and $\Theta_{\mathrm{TIR}}^{+}$.

\section{Axial Focusing, Rainbows, and Glories}

If a plane wave were traveling in the positive $z$ direction and incident on the sphere, the $p$ family of rays transmitted into the sphere would form a spherical aberration caustic with the paraxial focusing position ${ }^{27}$

$$
z=(-1)^{p} a /(2 p-1-N) .
$$

According to the reciprocity principle, ${ }^{15}$ if a point source inside the sphere is located at

$$
q_{\mathrm{AF}} \equiv 1 /(2 p-1-N),
$$

axial focusing of the $\alpha \ll 1$ paraxial rays occurs in the positive $z$ direction for odd $p$ and in the negative $z$ direction for even $p$ when $p>(N / 2)+1$. If $p<N / 2$, focusing of the $(\pi-\alpha) \ll 1$ paraxial rays occurs in the opposite direction, and for $N / 2<p<(N / 2)+1$, axial focusing of the $p$ ray family does not occur.

Both a rainbow and a glory are formed by $\alpha<\pi / 2$ members of the $p$ ray family when ${ }^{23} q>q_{\mathrm{AF}}$. This may be demonstrated as follows. Consider scattering of $\alpha$ $\ll 1$ paraxial rays for $q=q_{\mathrm{AF}}+\epsilon$ with $|\epsilon| \ll 1$. Equation (5) then reduces to

$$
\begin{aligned}
\Theta= & (p-1) \pi-\alpha\left(\epsilon / q_{\mathrm{AF}}\right)+\left(\alpha^{3} / 6\right)\left\{\left[1-q_{\mathrm{AF}}^{3}\right.\right. \\
& \left.\times\left(2 p-1-N^{3}\right)\right]+\left(\epsilon / q_{\mathrm{AF}}\right)\left[1-3 q_{\mathrm{AF}}^{3}\right. \\
& \left.\left.\times\left(2 p-1-N^{3}\right)\right]\right\}+O\left(\alpha^{5}\right) .
\end{aligned}
$$

As the source moves outward from the center of the sphere and $q$ increases through $q_{\mathrm{AF}}$, the term in Eq. (18) proportional to $\alpha$ changes sign from positive to negative while the term proportional to $\alpha^{3}$ remains positive. Thus $\Theta(\alpha)$ changes from being an increasing function of $\alpha$ to a function that first decreases, reaches a relative minimum at

$$
\alpha_{\mathrm{R}} \equiv\left(2 \epsilon / q_{\mathrm{AF}}\right)^{1 / 2}\left[1-q_{\mathrm{AF}}^{3}\left(2 p-1-N^{3}\right)\right]^{-1 / 2},
$$

corresponding to the rainbow deflection angle

$$
\Theta_{\mathrm{R}}=(p-1) \pi-\left(2 \epsilon / 3 q_{\mathrm{AF}}\right) \alpha_{\mathrm{R}},
$$

and then increases back through $(p-1) \pi$ at

$$
\alpha_{\mathrm{G}}=3^{1 / 2} \alpha_{\mathrm{R}}
$$

to produce glory scattering. As $q \rightarrow 1$, the deflection angle of the $p$ rainbow decreases to

$$
\Theta_{\mathrm{R}}=(p-1) \pi-2(p-1) \alpha+\sin ^{-1}[N \sin (\alpha)],
$$

with

$$
\cos (\alpha)=[2(p-1) / N]\left(N^{2}-1\right)^{1 / 2}\left[4(p-1)^{2}-1\right]^{-1 / 2} .
$$

\section{Scattering Resonances}

In ray theory, the far-zone scattered intensity diverges at glories and rainbows as a result of the vanishing of either $\sin (\theta)$ with $\alpha \neq 0$ or $\mathrm{d} \Theta / \mathrm{d} \alpha$, respectively, in the denominator of Eq. (7). For the type of resonances encountered in interior source scattering, the intensity becomes large but finite over a small range of $\theta$ because of $\mathrm{d} \Theta / \mathrm{d} \alpha$ becoming small but nonzero there. Physically, this is a focusing effect and corresponds to a scattering angle interval where the rays exiting the sphere deviate from each other only slightly. The resonance of the $p=1$ ray family for $N=4 / 3, q_{c}=0.75$, and $q=0.748$ is shown in Fig. 3.

A resonance of this type occurs for each $p$ ray family when $q=q_{c}-\epsilon$ with $\epsilon \ll 1$. The quantity $\mathrm{d} \Theta / \mathrm{d} \alpha$ reaches a minimum value of

$$
(\mathrm{d} \Theta / \mathrm{d} \alpha)_{\min }=3\left[N \epsilon /\left(N^{2}-1\right)\right]^{1 / 3}[(2 p-1) / 2]^{2 / 3}
$$

at the deflection angle

$$
\Theta_{\mathrm{RES}}=\Theta_{\mathrm{TIR}}^{0}-(2 p-1) N \epsilon /\left[4\left(N^{2}-1\right)^{1 / 2}\right]+O\left(\epsilon^{4 / 3}\right) .
$$

As $\epsilon \rightarrow 0$, the potential divergence in $I\left(\theta_{\mathrm{RES}}\right)$ is removed because the transmission angle of the resonant ray is

$$
\theta_{t}=\pi / 2-[2 N \epsilon /(2 p-1)]^{1 / 3}\left(N^{2}-1\right)^{1 / 6},
$$

which causes the Fresnel transmission coefficient of Eqs. (4) to approach zero at a faster rate.

These interior source resonances differ from MDRs in three fundamental ways. First, they correspond to a given number of internal reflections, whereas MDRs are a collective effect produced by all numbers of internal reflections considered together. Also, they occur over a small scattering angle interval, whereas MDRs occur at all angles. Last, the resonance mechanism is different. These resonances occur because the rays leaving the sphere in a small angular interval are nearly parallel and produce partial focusing, whereas MDRs occur when a totally internally reflecting ray advances in phase by an integer number of cycles on successive orbits beneath the sphere surface.

\section{E. Total Internal Reflection Rainbows}

A second rainbow is produced by $\alpha>\pi / 2$ members of the $p$ ray family when $q>q_{c}$ and occurs in the total internal reflection interval of relation (13) and Eq. (14). As $\alpha$ increases toward $\alpha_{\text {TIR }}^{-}$for $q>q_{c}$, the deflection angle $\Theta$ increases toward $\Theta_{\text {TIR }}^{-}$, approaching it with infinite slope. This is illustrated in Fig. 4 for $p=1, N=4 / 3$, and $q$ $=0.90$. As $\alpha$ then increases from $\alpha_{\text {TIR }}^{+}$to $\pi$, the deflection angle decreases from $\Theta_{\text {TIR }}^{+}$, initially with infinite slope, into the total internal reflection region of relation (13) and Eq. (14), has a relative minimum at the total internal reflection rainbow angle $\Theta_{\mathrm{TIRR}}$, and then increases 


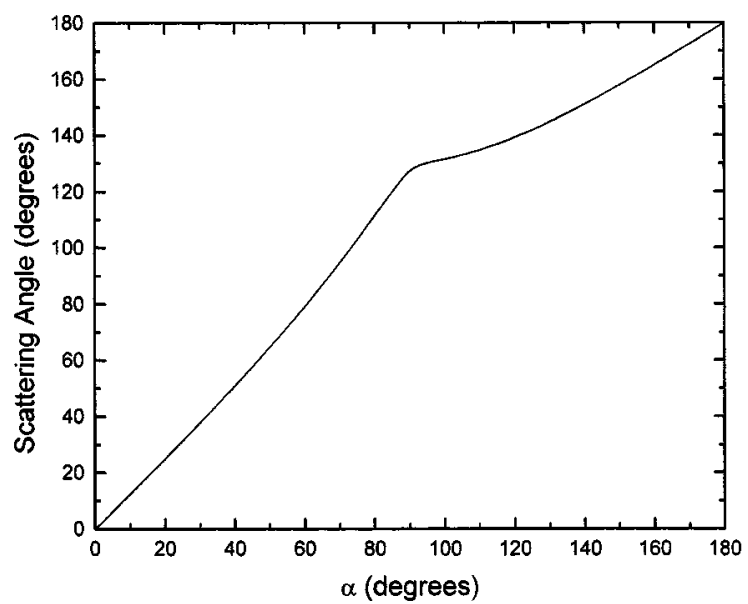

(a)

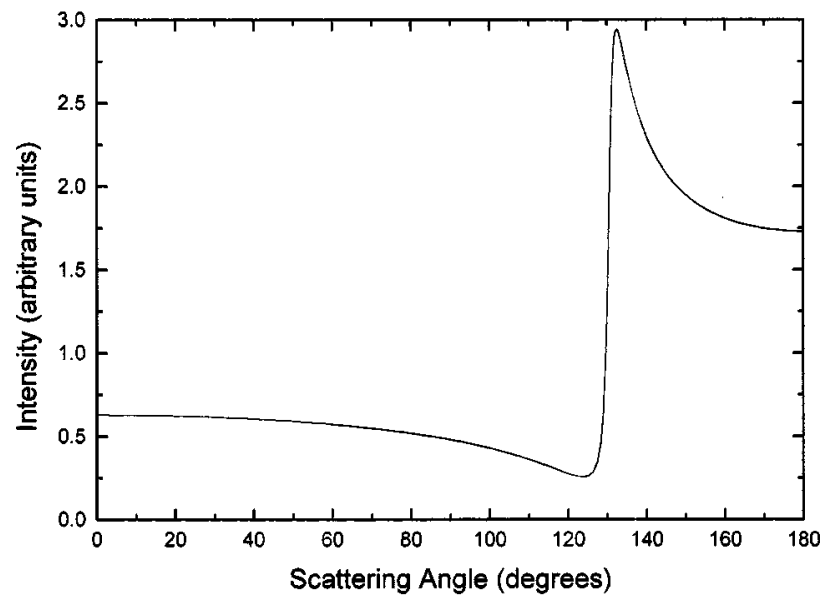

(b)

Fig. 3. (a) Scattering angle $\theta$ as a function of $\alpha$ of the $p=1$ ray family for $N=4 / 3$ and $q=0.748$ and (b) $p=1$ ray scattering intensity exhibiting the scattering resonance at $\theta \approx 132^{\circ}$.

back out of the total internal reflection region. For $q$ $=q_{c}+\epsilon$ with $\epsilon \ll 1$, the total internal reflection rainbow angle is found to be

$$
\begin{aligned}
\Theta_{\mathrm{TIRR}}= & \Theta_{\mathrm{TIR}}^{0}+(3 / 2)(N \epsilon)^{2 / 3}(2 p-1)^{1 / 3} \\
& \times\left(N^{2}-1\right)^{-1 / 6}+O(\epsilon),
\end{aligned}
$$

and as $q \rightarrow 1$, it increases to

$$
\Theta_{\mathrm{TIRR}}=p \pi-2 p \beta+\sin ^{-1}[N \sin (\beta)],
$$

with

$$
\begin{aligned}
\alpha & =\pi-\beta, \\
\cos (\beta) & =(2 p / N)\left(N^{2}-1\right)^{1 / 2}\left(4 p^{2}-1\right)^{-1 / 2} .
\end{aligned}
$$

In the $q \rightarrow 1$ limit, Eqs. (22), (23), (28), and (30) illustrate that the deflection angle of the $p+1$ rainbow and the $p$ total internal reflection rainbow coincide.

\section{F. Ray Scattering of an Isotropic Point Source in a Water Droplet}

To gain a better understanding of the rich variety of scattering phenomena that occur for different $p$ families of rays for an interior source, we consider the specific ex- ample of a spherical water droplet with $N=4 / 3$. We find that for each larger value of $p$, the scattered intensity exhibits new features not encountered for previous $p$. But at the same time, the scattered intensity of the $p$ ray family also becomes increasingly weak for larger $p$ as a result of the increasing number of Fresnel reflection coefficients in Eq. (7). Thus we consider the specific details of $\Theta(\alpha)$ for only $1 \leqslant p \leqslant 4$. For use in Section 3, the scattering angle associated with each deflection angle calculated in this subsection is given in parentheses.

The behavior of $\Theta(\alpha)$ for $p=1,2,3$, and 4 and $q>q_{c}$ is qualitatively illustrated in Figs. 4, 5, 6, and 7, respectively. From Eqs. (9) and (14), the critical source position is $q_{c}=0.75$, and the width of the total internal reflection region is identical for all $p$ ray families. Axial focusing of $p=1$ rays and the associated rainbow and glory do not occur for $N=4 / 3$. When the source is located at $q$ $=q_{c}=0.75$, total internal reflection of the $p=1$ rays commences at the deflection angle $\Theta_{\mathrm{TIR}}^{0}=131.41^{\circ}$. As the source moves along the $z$ axis from $q_{c}$ toward the sphere surface, the total internal reflection region ini-

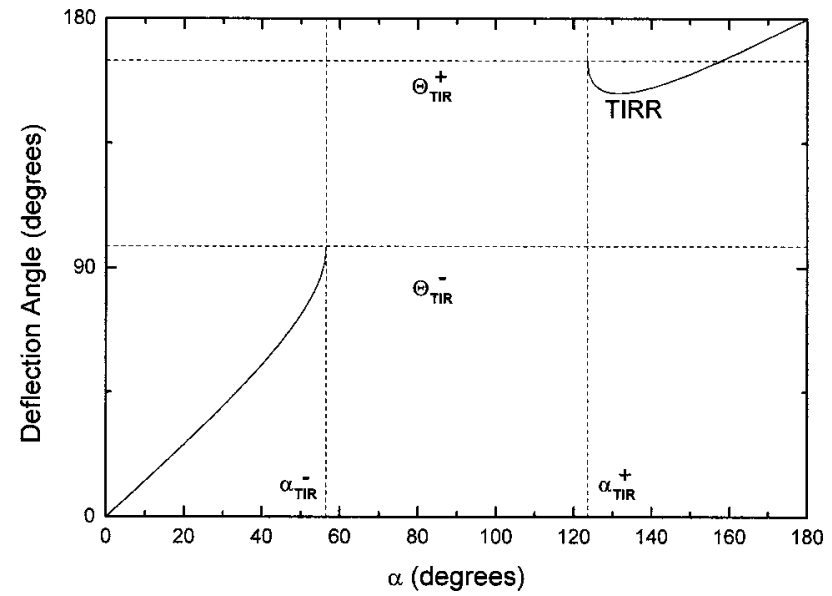

Fig. 4. Deflection angle of the $p=1$ ray family as a function of $\alpha$ for $N=4 / 3$ and $q=0.90$. The total internal reflection region extends from $\Theta_{\text {TIR }}^{-}$to $\Theta_{\text {TIR }}^{+}$, and the total internal reflection rainbow is indicated by TIRR.

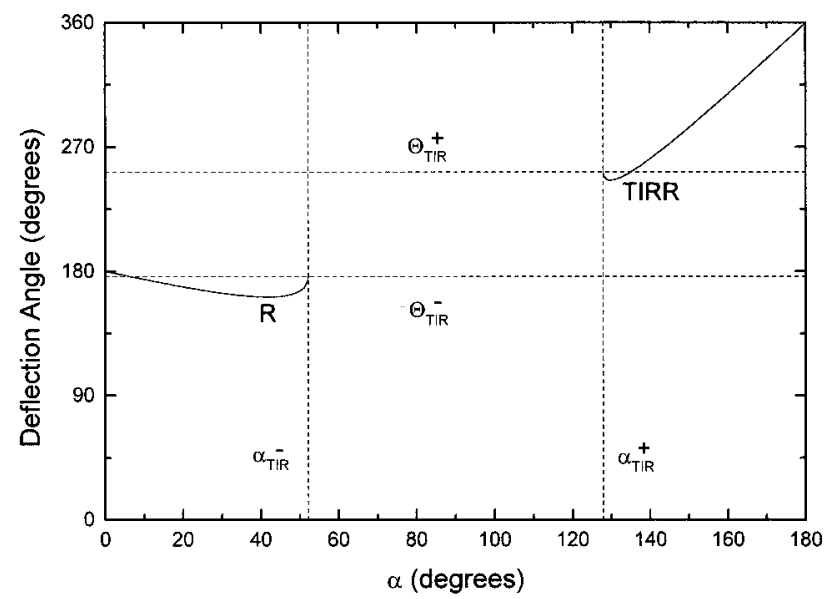

Fig. 5. Deflection angle $\Theta$ of the $p=2$ ray family as a function of $\alpha$ for $N=4 / 3$ and $q=0.95$. The rainbow is indicated by $\mathrm{R}$, and the total internal reflection rainbow is indicated by TIRR. A ray theory glory occurs at $\Theta=180^{\circ}$ for $q \leqslant 0.91$. 


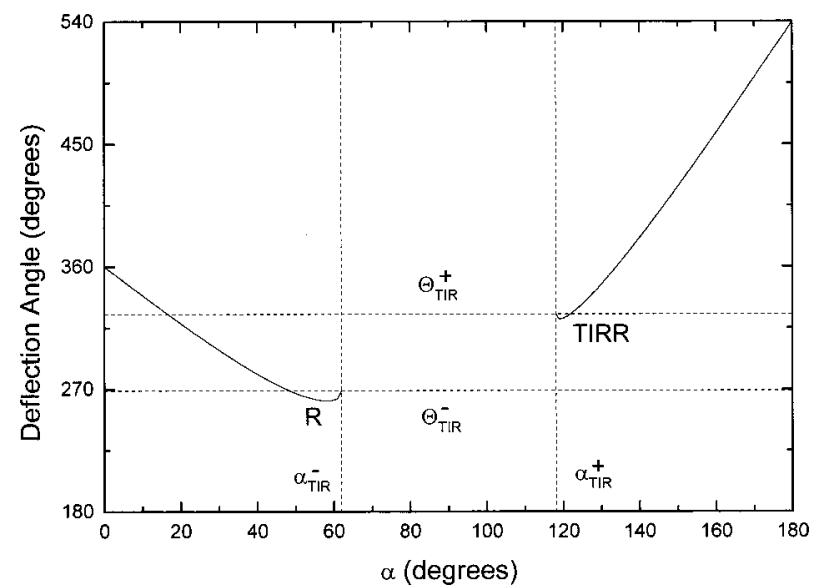

Fig. 6. Deflection angle $\Theta$ of the $p=3$ ray family as a function of $\alpha$ for $N=4 / 3$ and $q=0.85$. The rainbow is indicated by $\mathrm{R}$, and the total internal reflection rainbow is indicated by TIRR. Both $\alpha \ll 1$ paraxial rays and the $\alpha>90^{\circ}$ rays contributing to the total internal reflection rainbow extend into the total internal reflection region.

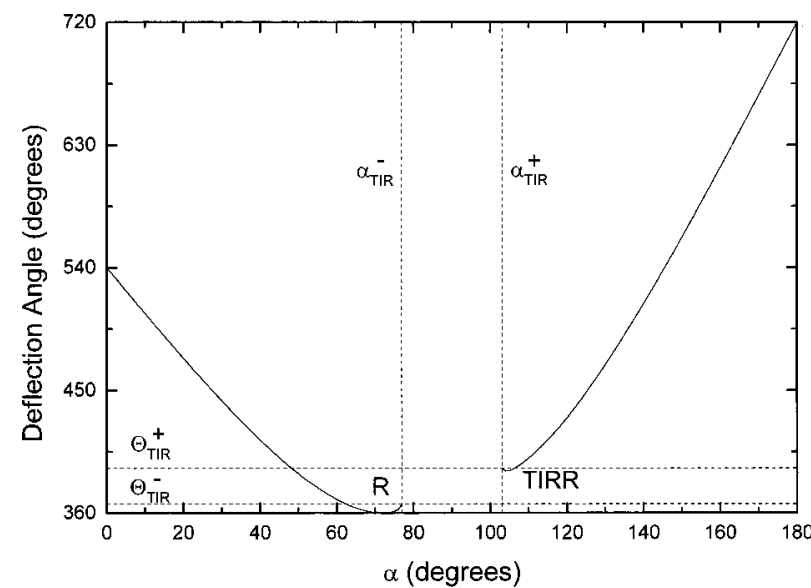

Fig. 7. Deflection angle $\Theta$ of the $p=4$ ray family as a function of $\alpha$ for $N=4 / 3$ and $q=0.77$, producing a rainbow-enhanced glory at $\Theta_{R}=360^{\circ}$. The rainbow is indicated by $R$, and the total internal reflection rainbow is indicated by TIRR. For $q$ $<0.77$, the rainbow angle is $\Theta_{\mathrm{R}}>360^{\circ}$. For $0.77<q$ $<0.80, \Theta_{\mathrm{R}}<360^{\circ}$ and the rainbow is accompanied by two glory rays. For $q>0.80, \Theta_{\mathrm{TIR}}^{-}<360^{\circ}$ and one of the glory rays ceases to exist in ray theory.

tially widens very rapidly, as was found in Eq. (15), and then widens at a slower rate as $q \rightarrow 1$. For example, when $q=0.80$, the total internal reflection region has grown to $111.05^{\circ} \leqslant \Theta \leqslant 151.77^{\circ}$, and for $q=0.99$ the region is $90.66^{\circ} \leqslant \Theta \leqslant 172.16^{\circ}$. As $q$ increases from 0.75 to 0.99 , the total internal reflection rainbow angle increases from $\Theta_{\mathrm{TIRR}}=131.41^{\circ}$ to $158.49^{\circ}$.

Axial focusing of $p=2$ rays occurs at $\Theta_{\mathrm{AF}}=180^{\circ}$ for $q=q_{\mathrm{AF}}=0.60$, and as was shown in Eqs. (18)-(21), a rainbow plus a $\Theta=180^{\circ}$ glory occurs for $q>0.60$. As $q$ increases from 0.60 to 0.99 , the rainbow angle decreases from $\Theta_{\mathrm{R}}=180^{\circ}$ to $159.47^{\circ}$. For $q=q_{c}=0.75$, total internal reflection commences at $\Theta_{\mathrm{TIR}}^{0}=214.23^{\circ} \quad(\theta$ $\left.=145.77^{\circ}\right)$. The total internal reflection region grows to $173.48^{\circ} \leqslant \Theta \leqslant 254.98^{\circ} \quad\left(105.02^{\circ} \leqslant \theta \leqslant 180.00^{\circ}\right)$ for $q=0.99$, folding over upon itself from $173.48^{\circ}$ to $180.00^{\circ}$. As is illustrated in Fig. 5 for $q=0.95$, the total internal reflection interval for $q \geqslant 0.91$ includes the deflection angle $180^{\circ}$, and so glory scattering at $\Theta=180^{\circ}$ ceases to occur for $q \geqslant 0.91$ in the context of ray theory. As $q$ increases from 0.75 to 0.99 , the total internal reflection rainbow angle increases from $\Theta_{\mathrm{TIRR}}=214.23^{\circ}$ $\left(145.77^{\circ}\right)$ to $248.57^{\circ}\left(111.43^{\circ}\right)$.

Axial focusing of $p=3$ rays occurs at $\Theta_{\mathrm{AF}}=360^{\circ}$ for $q=q_{\mathrm{AF}}=0.27$, and as $q$ increases from 0.27 to 0.99 , the rainbow angle decreases from $\Theta_{\mathrm{R}}=360^{\circ}\left(0^{\circ}\right)$ to $249.82^{\circ}$ $\left(110.18^{\circ}\right)$. For $q>q_{\mathrm{AF}}$, the rainbow is accompanied by a glory at $\Theta=360^{\circ}\left(0^{\circ}\right)$. Total internal reflection commences at $\Theta_{\mathrm{TIR}}^{0}=297.05^{\circ}\left(62.95^{\circ}\right)$ for $q=q_{c}=0.75$, and the total internal reflection region grows to $256.30^{\circ}$ $\leqslant \Theta \leqslant 337.80^{\circ}\left(22.20^{\circ} \leqslant \theta \leqslant 103.70^{\circ}\right)$ for $q=0.99$. As $q$ increases from 0.75 to 0.99 , the total internal reflection rainbow angle increases from $\Theta_{\mathrm{TIRR}}=297.05^{\circ}$ $\left(62.95^{\circ}\right)$ to $333.57^{\circ}\left(26.43^{\circ}\right)$. In Fig. 6 for $q=0.85$, the deflection angle of the $\alpha<\pi / 2$ rays decreases through the total internal reflection region before reaching a minimum at the rainbow angle $\Theta_{\mathrm{R}}$. As a result, the $p=3$ intensity in the total internal reflection interval contains interference of $\alpha<\pi / 2$ rays with $\alpha>\pi / 2$ rays.

For $p=4$, axial focusing occurs at $\Theta_{\mathrm{AF}}=540^{\circ}\left(180^{\circ}\right)$ for $q=q_{\mathrm{AF}}=0.18$, and as $q$ increases from $q=q_{\mathrm{AF}}$ $=0.18$ to 0.77 , the rainbow deflection angle decreases from $\Theta_{\mathrm{R}}=540^{\circ}\left(180^{\circ}\right)$ to $360^{\circ}\left(0^{\circ}\right)$. For $q=0.77$, the rainbow minimum at $360^{\circ}$ produces the rainbowenhanced glory ${ }^{24}$ indicated in Fig. 7. For $0.77<q$ $<0.99$, the rainbow angle further decreases from $\Theta_{R}$ $=360^{\circ}\left(0^{\circ}\right)$ to $334.85^{\circ}\left(25.15^{\circ}\right)$ and is accompanied by two different $\Theta=360^{\circ}$ glory rays $^{24}$ for $0.77<q<0.80$. For $q=q_{c}=0.75$, total internal reflection commences at $\Theta_{\mathrm{TIR}}^{0}=379.87^{\circ}\left(19.87^{\circ}\right)$. The total internal reflection region grows to $339.12^{\circ} \leqslant \Theta \leqslant 420.62^{\circ} \quad\left(0.00^{\circ} \leqslant \Theta\right.$ $\leqslant 60.62^{\circ}$ ) for $q=0.99$, folding over upon itself from $0.00^{\circ}$ to $20.88^{\circ}$. For $q \geqslant 0.80$, the angle $\Theta=360^{\circ}$ is included in the total internal reflection region, and one of the two glory rays that accompanied the rainbow no longer occurs in the context of ray theory. As $q$ increases from 0.75 to 0.99 , the total internal reflection rainbow angle increases from $\Theta_{\mathrm{TIRR}}=379.87^{\circ}\left(19.87^{\circ}\right)$ to $417.46^{\circ}$ $\left(57.46^{\circ}\right)$. Last, the values of $\Theta_{\mathrm{R}}$ for the $p+1$ rainbow and $\Theta_{\text {TIRR }}$ for the $p$ total internal reflection rainbow for $q=0.99$ are nearly equal and approach the $q \rightarrow 1$ prediction of $\Theta=158.99^{\circ}, 249.19^{\circ}, 334.21^{\circ}$, and $418.10^{\circ}$ for $p=1,2,3,4$ from opposite directions.

\section{WAVE SCATTERING OF AN INTERIOR ELECTRIC DIPOLE SOURCE}

\section{A. Scattered Fields, Debye Series Expansion, and Orientation-Averaged Intensity}

Comparison of the ray theory predictions of Section 2 with the features of the exact wave-scattered intensity is not especially straightforward and must be pursued in a number of stages. First, if an oscillating electric dipole source of unit strength is located at $z_{0}$ on the positive $z$ axis and the dipole vector points in the $x$ direction, the far-zone scattered electric field simplifies from the results of Refs. $10-16$ to 


$$
\begin{aligned}
\mathbf{E}_{\mathrm{scatt}}(r, \theta, \phi)= & i[\exp (i k r) /(k r)] \\
& \times\left[T_{1}(\theta) \cos (\phi) \mathbf{u}_{\theta}-T_{2}(\theta) \sin (\phi) \mathbf{u}_{\phi}\right]
\end{aligned}
$$

where

$$
\begin{aligned}
T_{1}(\theta)= & \sum_{n=1}^{\infty}(-i)^{n}\left\{c _ { n } \left[j_{n+1}\left(N k z_{0}\right) /\right.\right. \\
& \left.(n+1)-j_{n-1}\left(N k z_{0}\right) / n\right] \tau_{n, 1}(\theta)+i d_{n} \\
& \left.\times[(2 n+1) / n(n+1)] j_{n}\left(N k z_{0}\right) \pi_{n, 1}(\theta)\right\}, \\
T_{2}(\theta)= & \sum_{n=1}^{\infty}(-i)^{n}\left\{c _ { n } \left[j_{n+1}\left(N k z_{0}\right) /\right.\right. \\
& \left.(n+1)-j_{n-1}\left(N k z_{0}\right) / n\right] \pi_{n, 1}(\theta)+i d_{n} \\
& \left.\times[(2 n+1) / n(n+1)] j_{n}\left(N k z_{0}\right) \tau_{n, 1}(\theta)\right\} .
\end{aligned}
$$

The free-space wave number of the electric dipole radiation is

$$
k=2 \pi / \lambda,
$$

and the partial wave scattering amplitudes are

$$
\begin{aligned}
c_{n}= & {\left[i / N(k a)^{2}\right]\left[h_{n}^{(1)}(k a) j_{n}^{\prime}(N k a)\right.} \\
& -N h_{n}^{(1) \prime}(k a) j_{n}(N k a)-\left(N^{2}-1\right) \\
& \left.\times h_{n}^{(1)}(k a) j_{n}(N k a) /(N k a)\right]^{-1}, \\
d_{n}= & {\left[i / N(k a)^{2}\right]\left[N h_{n}^{(1)}(k a)\right.} \\
& \left.\times j_{n}^{\prime}(N k a)-h_{n}^{(1) \prime}(k a) j_{n}(N k a)\right]^{-1},
\end{aligned}
$$

where $j_{n}$ are spherical Bessel functions, $h_{n}^{(1)}$ are spherical Hankel functions of the first kind, the angular functions are

$$
\pi_{n, m}(\theta)=P_{n, m}(\theta) / \sin (\theta), \quad \tau_{n, m}(\theta)=(\mathrm{d} / \mathrm{d} \theta) P_{n, m}(\theta),
$$

and $P_{n, m}(\theta)$ are associated Legendre polynomials. If the oscillating electric dipole vector points in the $y$ direction, the scattered electric field is

$$
\begin{aligned}
\mathbf{E}_{\text {scatt }}(r, \theta, \phi)= & i[\exp (i k r) /(k r)] \\
& \times\left[T_{1}(\theta) \sin (\phi) \mathbf{u}_{\theta}+T_{2}(\theta) \cos (\phi) \mathbf{u}_{\phi}\right],
\end{aligned}
$$

and if it points in the $z$ direction, the scattered electric field is

$$
\mathbf{E}_{\text {scatt }}(r, \theta, \phi)=-i[\exp (i k r) /(k r)] T_{3}(\theta) \mathbf{u}_{\theta},
$$

where

$$
T_{3}(\theta)=\sum_{n=1}^{\infty}(-i)^{n}(2 n+1) c_{n}\left[j_{n}\left(N k z_{0}\right) /\left(N k z_{0}\right)\right] \tau_{n, 0}(\theta) .
$$

The wave scattering intensity for a given dipole orientation is

$$
I_{\text {scatt }}(r, \theta, \phi)=\left(1 / 2 \mu_{0} c\right)\left|\mathbf{E}_{\text {scatt }}(r, \theta, \phi)\right|^{2},
$$

where $\mu_{0}$ is the permeability of free space and $c$ is the speed of light.
If the interior electric dipole source is a fluorescing molecule in a liquid droplet, the rotational diffusion time of the molecule is usually much shorter than the fluorescent transition deexcitation lifetime. In this case, the scattered intensity is averaged over all electric dipole orientations. When Eqs. (31), (32), and (36-38) are substituted into Eq. (39), the average over dipole orientations can be performed analytically, ${ }^{16,28}$ giving

$$
\begin{aligned}
I_{\text {scatt }}^{\text {ave }}(r, \theta)= & \left(1 / 2 \mu_{0} c\right)\left[\left|T_{1}(\theta)\right|^{2}+\left|T_{2}(\theta)\right|^{2}\right. \\
& \left.+\left|T_{3}(\theta)\right|^{2}\right] /\left(3 k^{2} r^{2}\right) .
\end{aligned}
$$

Since the direction of the oscillating dipole is averaged over all directions, the orientation-averaged intensity of Eq. (40) in the $a \gg \lambda$ limit is expected to bear a strong resemblance to the ray scattering intensity for an isotropic source.

The ray intensity of Eqs. (7) and (8), however, describes scattering by a single $p$ ray family, whereas the partial wave scattering amplitudes of Eqs. (34) include the combined effects of all $p$ families of scattered waves. The partial wave amplitudes for scattering of a single $p$ wave family are obtained from $c_{n}$ and $d_{n}$ by using the Debye series decomposition. The partial wave scattering amplitudes may be written as a sum of transmitted transverse magnetic (TM) and transverse electric (TE) waves, $T_{n}^{\mathrm{TM}}$ and $T_{n}^{\mathrm{TE}}$, and waves transmitted following an arbitrary number of TM and TE internal reflections, $R_{n}^{\mathrm{TM}}$ and $R_{n}^{\mathrm{TE}}$, i.e.,

$$
\begin{aligned}
& c_{n}=-T_{n}^{\mathrm{TM}} /\left(1-R_{n}^{\mathrm{TM}}\right)=-\sum_{p=1}^{\infty}\left(R_{n}^{\mathrm{TM}}\right)^{p-1} T_{n}^{\mathrm{TM}}, \\
& d_{n}=-T_{n}^{\mathrm{TE}} /\left(1-R_{n}^{\mathrm{TE}}\right)=-\sum_{p=1}^{\infty}\left(R_{n}^{\mathrm{TE}}\right)^{p-1} T_{n}^{\mathrm{TE}} .
\end{aligned}
$$

The partial-wave transmission and reflection amplitudes are found to be

$$
\begin{aligned}
T_{n}^{\mathrm{TM}}=[ & \left.-2 i / N(k a)^{2}\right]\left[h_{n}^{(1)}(k a) h_{n}^{(2)^{\prime}}(N k a)\right. \\
& -N h_{n}^{(1) \prime}(k a) h_{n}^{(2)}(N k a)-\left(N^{2}-1\right) \\
& \left.\times h_{n}^{(1)}(k a) h_{n}^{(2)}(N k a) /(N k a)\right]^{-1}, \\
T_{n}^{\mathrm{TE}}=[ & \left.-2 i / N(k a)^{2}\right]\left[N h_{n}^{(1)}(k a) h_{n}^{(2) \prime}(N k a)\right. \\
& \left.-h_{n}^{(1) \prime}(k a) h_{n}^{(2)}(N k a)\right]^{-1}, \\
R_{n}^{\mathrm{TM}}=- & {\left[h_{n}^{(1)}(k a) h_{n}^{(1) \prime}(N k a)-N h_{n}^{(1) \prime}(k a) h_{n}^{(1)}(N k a)\right.} \\
& \left.-\left(N^{2}-1\right) h_{n}^{(1)}(k a) h_{n}^{(1)}(N k a) /(N k a)\right] / \\
& {\left[h_{n}^{(1)}(k a) h_{n}^{(2) \prime}(N k a)-N h_{n}^{(1) \prime}(k a) h_{n}^{(2)}(N k a)\right.} \\
& \left.-\left(N^{2}-1\right) h_{n}^{(1)}(k a) h_{n}^{(2)}(N k a) /(N k a)\right], \\
R_{n}^{\mathrm{TE}}=- & {\left[N h_{n}^{(1)}(k a) h_{n}^{(1) \prime}(N k a)\right.} \\
& \left.-h_{n}^{(1) \prime}(k a) h_{n}^{(1)}(N k a)\right] / \\
{[} & \left.N h_{n}^{(1)}(k a) h_{n}^{(2) \prime}(N k a)-h_{n}^{(1) \prime}(k a) h_{n}^{(2)}(N k a)\right],
\end{aligned}
$$

where $h_{n}^{(2)}$ are spherical Hankel functions of the second kind. The scattered field of the $p$ wave family is obtained 
by substituting the $p$ term of Eqs. (41) into Eqs. (32) and (38) in place of the full partial wave scattering amplitudes of Eqs. (34).

Numerical computation of Eqs. (32) and (38) proceeds as in Mie theory with one exception. The partial wave scattering amplitudes $c_{n}$ and $d_{n}$ of Eqs. (34) are more slowly convergent as a function of the partial wave number $n$ than are the partial wave scattering amplitudes $a_{n}$ and $b_{n}$ of Mie theory. This is due to the Debye series decomposition of $a_{n}$ and $b_{n}$ containing the product of two transmission amplitudes, each of which decreases $\mathrm{as}^{7}$ $\exp \left[-(2 \gamma)^{3 / 2} / 3\right]$ for $n=x+\gamma x^{1 / 3}$ and $n \gg x$, where the sphere size parameter is

$$
x \equiv k a,
$$

whereas Eqs. (41) contain only one such term. As a result, the highest partial wave employed in the numerical computations of Subsection 3.B is

$$
n_{\max }=1+x+20 x^{1 / 3} .
$$

\section{B. Wave Scattering of an Electric Dipole Source in a Water Droplet}

In Section 2, we considered an isotropic point source in ray theory, whereas in wave theory, the simplest source is an oscillating electric dipole whose radiation pattern is nonisotropic. But in the plane perpendicular to the dipole direction, the emitted radiation is both isotropic and TE polarized. Thus if the electric dipole vector points in the $x$ direction and the scattered intensity is examined in the $y z$ plane, i.e., $\phi=\pi / 2$, one would expect that the wave scattering intensity of Eqs. (31)-(39) and (41)-(45) for a $\gg \lambda$ should be well approximated by the isotropic pointsource ray intensity of Eqs. (7) and (8).

To verify this claim, we compared the $p=1$ ray scattering intensity for $N=4 / 3$ with the $p=1$ wave scattering intensity for an $x$-direction electric dipole source for $\phi=\pi / 2, \lambda=0.6328 \mu \mathrm{m}, a=30.0 \mu \mathrm{m}$, and $N=4 / 3$. The radius of the water droplet is in the semiclassical wave scattering regime, where ray theory is expected to be a good approximation to wave theory. The Debye series partial-wave amplitudes of Eqs. (41) were used to obtain the scattered intensity for the $p=1$ wave family. The results are shown in Figs. 8(a), 8(b), and 8(c) for the source positions $q=0.50,0.748$, and 0.99 , respectively. In each case, the wave intensity at $\theta=0^{\circ}$ was normalized to the $\theta=0^{\circ}$ ray intensity. In Fig. 8(a) for $q$ $=0.50$, the $p=1$ intensity is a relatively featureless, slowly increasing function of $\theta$. The ray and wave intensities are virtually identical, and the slow increase in the intensity is due to the gradual decrease in $|\mathrm{d} \Theta / \mathrm{d} \alpha|$.

Figure 8(b) for $p=1$ and $q=0.748$ exhibits the scattering resonance. The ray and wave intensities are in general agreement in the resonance region and are virtually identical below it. But the wave scattering intensity possesses an oscillatory structure for $140^{\circ} \lesssim \theta \lesssim 170^{\circ}$ that is characteristic of wave interference. This is not predicted in ray theory, since $\Theta(\alpha)$ monotonically increases from $0^{\circ}$ to $180^{\circ}$, and there is no two-ray region of $\theta$ where interference would occur. The oscillatory struc-

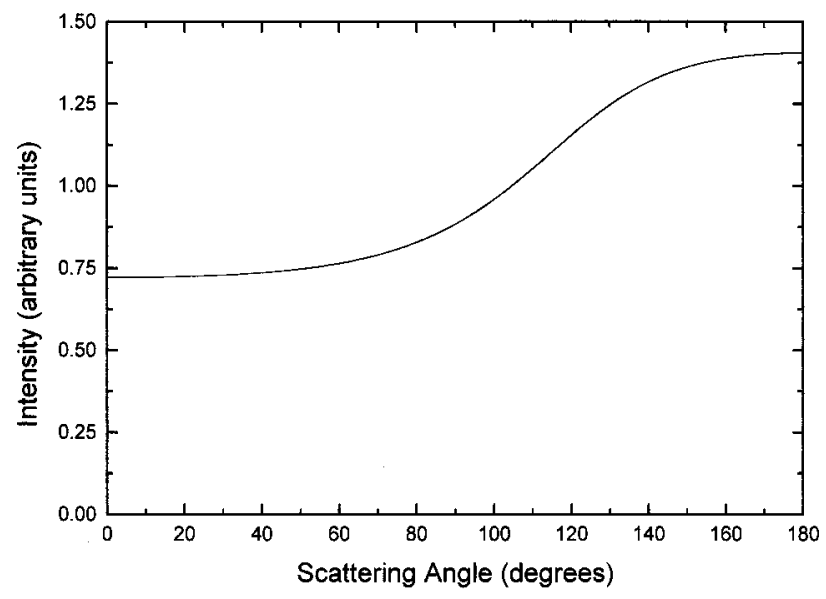

(a)

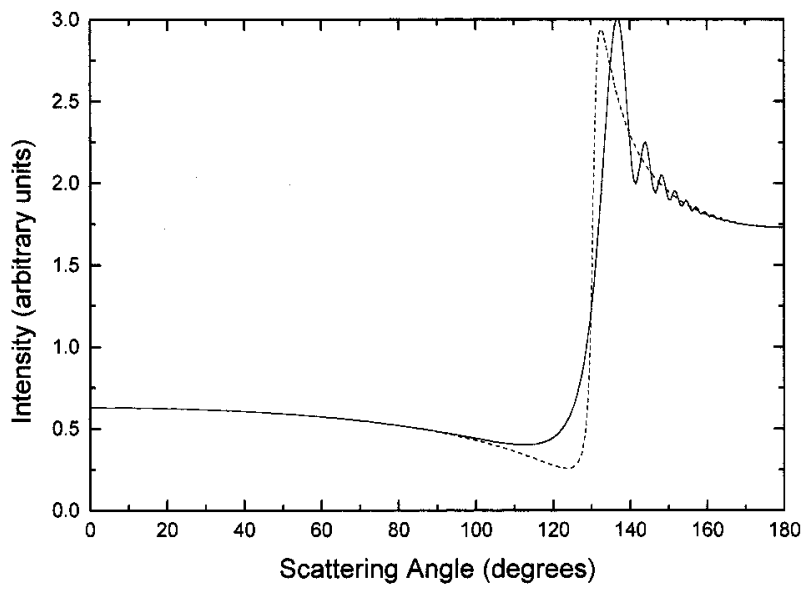

(b)

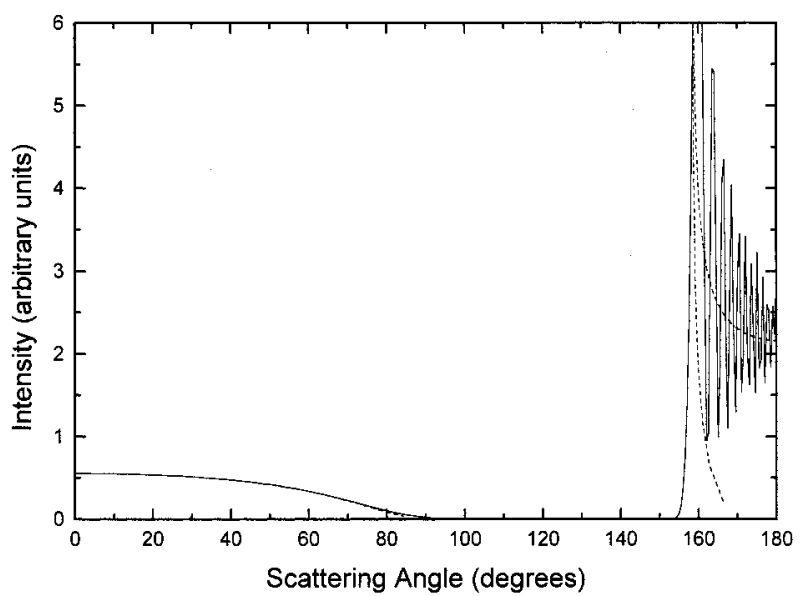

(c)

Fig. 8. Ray scattering intensity (dashed curves) for $N=4 / 3$ and $p=1$, and the wave-scattered intensity (solid curves) as a function of $\theta$ for $\phi=\pi / 2$ for an electric dipole source pointing in the $x$ direction with $\lambda=0.6328 \mu \mathrm{m}, a=30.0 \mu \mathrm{m}, N=4 / 3$, and $p=1$ for (a) $q=0.50$, (b) $q=0.748$, and (c) $q=0.99$. The ray and wave intensities are normalized to the same value at $\theta=0^{\circ}$.

ture in Fig. 8(b) is caused by the interference of the transmitted ray with radiation shed by electromagnetic surface waves produced by the incidence of the $p=1$ ray family 
at the sphere surface just below the critical angle for total internal reflection. This mechanism is illustrated in Fig. 9. Electromagnetic surface waves are produced by partial waves $n$ in the so-called edge region ${ }^{7,8} x-x^{1 / 3} \leqslant n$ $\lesssim x+x^{1 / 3}$. For $a=30.0 \mu \mathrm{m}$ and $q=0.748, p=1$ rays in the interval $78.58^{\circ} \leqslant \alpha \leqslant 101.42^{\circ}$ strike the sphere surface within the edge region and create electromagnetic surface waves.

Last, in Fig. 8(c) for $q=0.99$, both the total internal reflection region and the total internal reflection rainbow are clearly evident. Again, there is good agreement between the ray and wave intensities below the total internal reflection rainbow region, and the intensity in the supernumerary region oscillates back and forth about the ray theory intensity. Thus the scattering resonance/total internal reflection rainbow has the following morphology as a function of $q$. As $q$ increases toward $q_{c}$, the increase in $I(\theta)$ seen in Fig. 8(a) for $100 \leqq \theta \leqq 140^{\circ}$ evolves into the scattering resonance of Fig. 8(b). As $q$ increases beyond $q_{c}$, the $p=1$ scattering resonance and the oscillatory structure adjacent to it evolve into the $p=1$ total internal reflection rainbow and its supernumerary interference pattern of Fig. 8(c).

These results demonstrate that wave scattering in the plane perpendicular to the oscillating electric dipole for $a \gg \lambda$ is well described by ray scattering of an isotropic point source. The level of agreement between ray theory and wave theory for $a=30 \mu \mathrm{m}$ is especially encouraging, since for $q=0.99$ the source is only $0.3 \mu \mathrm{m}$ from the sphere surface, violating the semiclassical requirement that all distances involved in the problem be much larger than the wavelength of light.

Based on this close agreement for $p=1$, the wavescattered intensity was also computed for an electric dipole source in the $x$ direction as a function of $\theta$ for $\phi$ $=\pi / 2, \lambda=0.6328 \mu \mathrm{m}, a=30.0 \mu \mathrm{m}, N=4 / 3$, and $q=0.99$, for $p=2,3,4$, again by using the appropriate Debye series partial-wave amplitudes. The results, along with the $p=1$ intensity of Fig. 8(c) replotted on the same scale as that for $p=2,3,4$, are shown in Fig. 10 . The rainbow angle $\theta_{\mathrm{R}}$, the total internal reflection region, and the total internal reflection rainbow angle $\theta_{\text {TIRR }}$ calculated by using ray theory in Subsection 2.F are indicated in the plots.

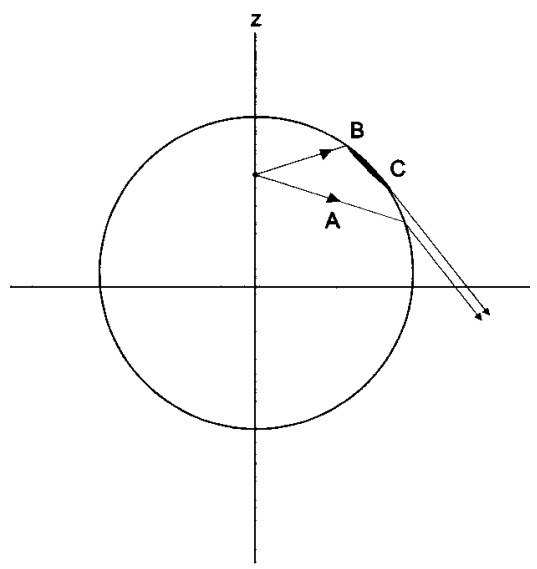

Fig. 9. Interference of $p=1$ rays (A) with the radiation shed by $p=1$ electromagnetic surface waves $(\mathrm{B}, \mathrm{C})$ in the vicinity of $\Theta_{\mathrm{TIR}}^{0}$ for $q \leqslant q_{c}$.
In addition to the semiclassical scattering phenomena examined in Section 2, Fig. 10 exhibits diffraction effects that smooth all the transitions between lit and unlit scattering regions. ${ }^{9}$ For example, for each value of $p$, the radiation shed by electromagnetic surface waves extends the $a<\pi / 2$ rainbow's supernumerary interference pattern beyond the deflection angle limit imposed by ray theory. This effect also occurs for Mie scattering of a plane wave by a spherical particle. ${ }^{29}$ In Mie scattering, the impact parameter of the $p$ rainbow ray component of the incident plane wave is near grazing incidence, and the rainbow ray exits the sphere at a relative minimum $\Theta_{R}$ of the deflection angle. The rainbow's supernumerary interference pattern occurs for $\Theta>\Theta_{R}$ and is produced by the interference of rays incident on the sphere to either side of the rainbow ray. The interference is limited in ray theory to the interval $\Theta_{\mathrm{R}} \leqslant \Theta \leqslant \Theta_{c}$, where $\Theta_{c}$ is the deflection angle of the grazing incident ray. But radiation shed by electromagnetic surface waves extends into the $\Theta>\Theta_{c}$ region and continues the supernumerary interference pattern to these larger deflection angles. The supernumerary pattern gradually damps out for $\Theta>\Theta_{c}$, since the amplitude of the surface wave radiation dies off exponentially as a function of $\Theta-\Theta_{c}$.

Similarly, for scattering by an interior source, the $p$ rainbow of Subsection 2.C occurs for $\Theta_{\mathrm{R}}<\Theta_{\mathrm{TIR}}^{-}$(see Figs. $5-7)$. The complex ray ${ }^{30}$ contribution to the rainbow extends away from the total internal reflection region and dies off faster than exponentially as a function of $\Theta_{R}$ $-\Theta$. According to ray theory, the supernumerary pattern should occur only for $\Theta_{\mathrm{R}} \leqslant \Theta \leqslant \Theta_{\text {TIR }}^{-}$. But radiation shed by electromagnetic surface waves begins at $\Theta_{\text {TIR }}^{-}$ and extends into the total internal reflection region, dying off exponentially as a function of $\Theta-\Theta_{\mathrm{TIR}}^{-}$. Again, it continues the supernumerary pattern into the $\Theta>\Theta_{\mathrm{TIR}}^{-}$ region. This is evident in Fig. 10(c) for $p=3$ but is obscured in Figs. 10(b) and 10(d) for $p=2$ and 4, respectively, because of the folding over of the total internal reflection region. The total internal reflection rainbow occurs for $\Theta_{\mathrm{TIRR}}<\Theta_{\mathrm{TIR}}^{+}$(see Figs. 4-7), and its complex ray contribution extends into the total internal reflection region. Again, according to ray theory, the supernumerary pattern should occur only for $\Theta_{\text {TIRR }} \leqslant \Theta \leqslant \Theta_{\text {TIR }}^{+}$. But radiation shed by electromagnetic surface waves begins at $\Theta_{\text {TIR }}^{+}$, extends away from the total internal reflection region, and continues the supernumerary pattern to $\Theta>\Theta_{\text {TIR }}^{+}$. This is evident in Figs. 10(a), 10(b), and 10(d) for 1,2 , and 4, respectively. In Fig. 10(c) for $p=3$, the supernumeraries are obscured by the $\theta=0^{\circ}$ glory.

In Fig. 10(a) for $p=1$, the lower limit of the total internal reflection region at $\Theta_{\mathrm{TIR}}^{-}$is smoothed by radiation shed by electromagnetic surface waves, and the upper limit at $\Theta_{\mathrm{TIR}}^{+}$is smoothed by the complex ray of the total internal reflection rainbow, which dominates over the radiation shed by the $\Theta>\Theta_{\text {TIR }}^{+}$surface waves beginning on the other side of the sphere at $\theta=187.84^{\circ}$. As this $\Theta$ $>\Theta_{\mathrm{TIR}}^{+}$surface wave radiation crosses $\theta=180^{\circ}$, it forms a surface wave glory.

In Fig. 10(b) for $p=2$, the $\Theta<\Theta_{\mathrm{TIR}}^{-}$surface waves beginning on the other side of the sphere at $\theta=186.52^{\circ}$ cross over the back, continue the $\theta=180^{\circ}$ glory that was 
extinguished in ray theory for $q>0.91$, interfere with the rainbow supernumeraries for $\theta \geqslant 170^{\circ}$, interfere with the complex ray of the rainbow at $\theta \approx 155^{\circ}$, and dominate the $p=2$ scattered intensity for $138^{\circ} \leqq \theta$ $\$ 155^{\circ}$. The surface waves provide a background contribution on which the $p=2$ rainbow is superimposed that resembles the real ray background contribution to the $p$ $=2$ rainbow for plane-wave incidence on a sphere with refractive index $n \lesssim 2$ and the real ray background contribution for $p=2$ rainbow scattering by a tilted cylinder $^{31}$ just below the rainbow merging transition. ${ }^{32}$ Last, the right-hand side of the total internal reflection region in Fig. 10(b) is smoothed by the complex ray of the rainbow rather than by the $\Theta<\Theta_{\text {TIR }}^{-}$surface wave radiation due to the folding over of the total internal reflection region.

The total internal reflection region for $p=3$ in Fig. 10 (c) is filled in by the interference of $\alpha<\pi / 2$ rays with $\alpha>\pi / 2$ rays as predicted in Fig. 6 . The featureless lowintensity region for $\theta \geqslant 130^{\circ}$ is due to scattering of $(\pi$ $-\alpha) \ll 1$ paraxial rays, and the enhancement at $\theta$ $\approx 0^{\circ}$ is the ray scattering glory of Fig. 6 . For $p=4$, the rainbow and the total internal reflection rainbow in Fig. 10(d) appear adjacent to each other as a result of the fold- ing over of the total internal reflection region. Ray scattering glories occur at $\theta=0^{\circ}$ and $180^{\circ}$, and an additional surface wave glory occurs at $\theta=0^{\circ}$. This surface wave glory is the continuation of the second $\theta=0^{\circ}$ glory that was extinguished in ray theory for $q>0.80$. Though not evident in Fig. 10, repeated Goos-Hanchen shifts ${ }^{33,34}$ at the critical angle for total internal reflection cause $\Theta_{\mathrm{TIR}}^{-}$ and $\Theta_{\text {TIR }}^{+}$to slowly increase above the prediction of Eq. (14) for large $p$.

In Fig. 11(a), the $1 \leqslant p \leqslant 5$ wave scattering intensities of Fig. 10 are displayed together. The scattering angle of the various $p$ rainbows and $p+1$ total internal reflection rainbows are seen to nearly coincide. Since the interval $120^{\circ} \lesssim \theta \leqq 150^{\circ}$ lies within the total internal reflection regions for $p=1,2$ and the low-intensity regions of $p$ $=3,4$, the dominant scattering mechanism is the $p$ $=5$ total internal reflection rainbow along with the $p=6$ rainbow, which is not shown in the figure.

To determine the importance of interference between the different $p$ wave families, the total $\phi=\pi / 2$ scattered intensity for an $x$-direction electric dipole source, obtained by using the full partial wave scattering amplitudes of Eqs. (34), is shown in Fig. 11(b). The $p=1,2$ total internal reflection rainbows at $\theta \approx 160^{\circ}$ and $\theta \approx 110^{\circ}$,

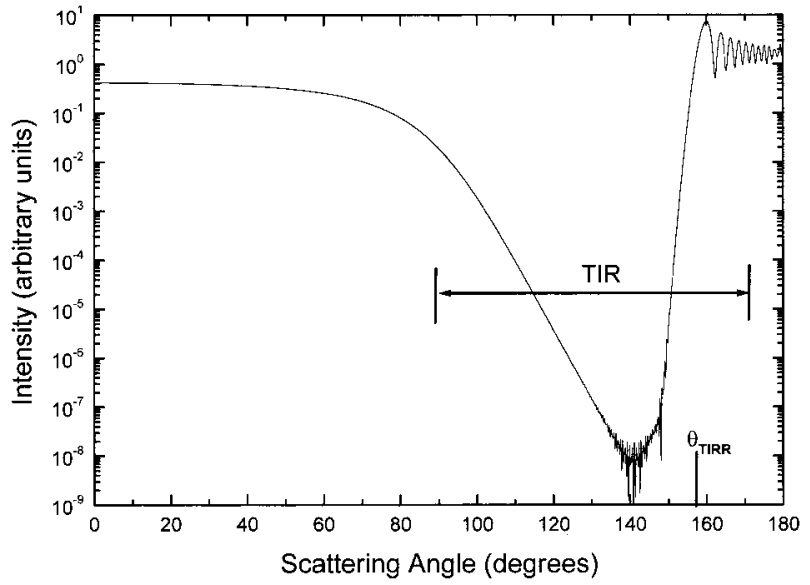

(a)

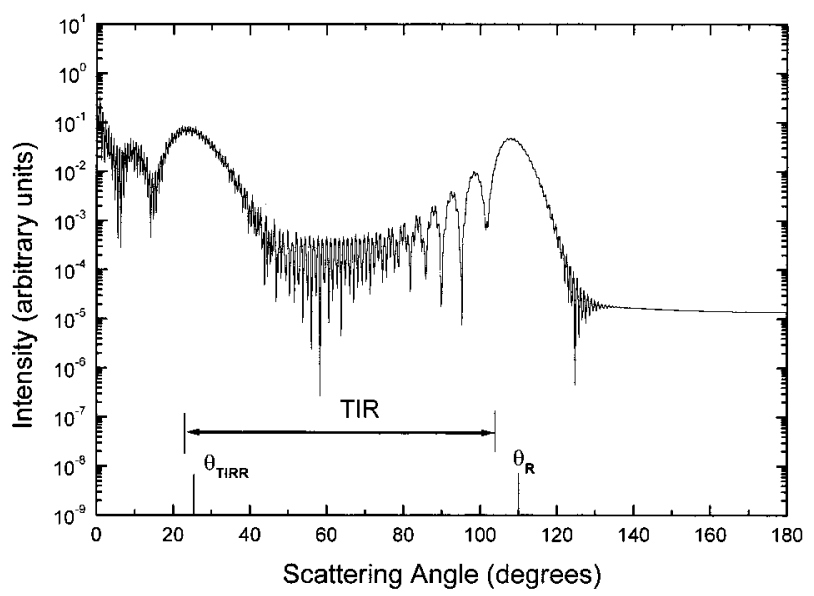

(c)

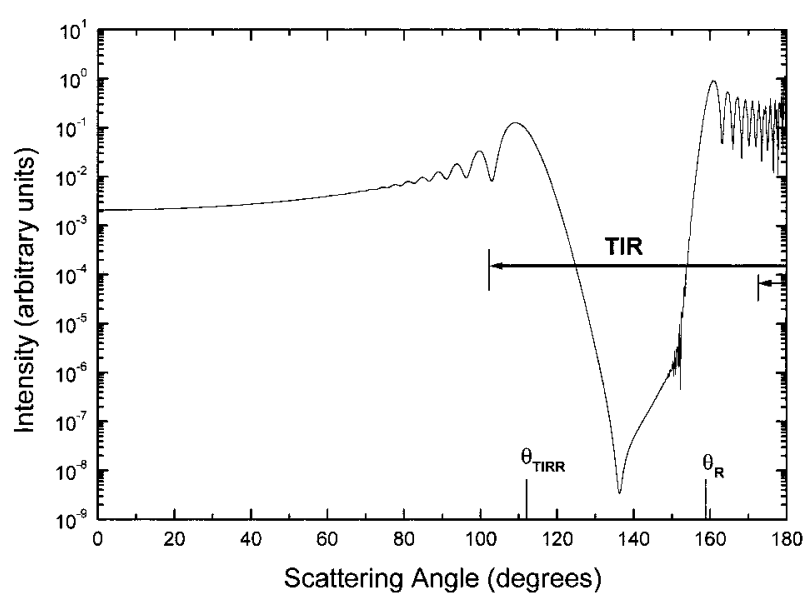

(b)

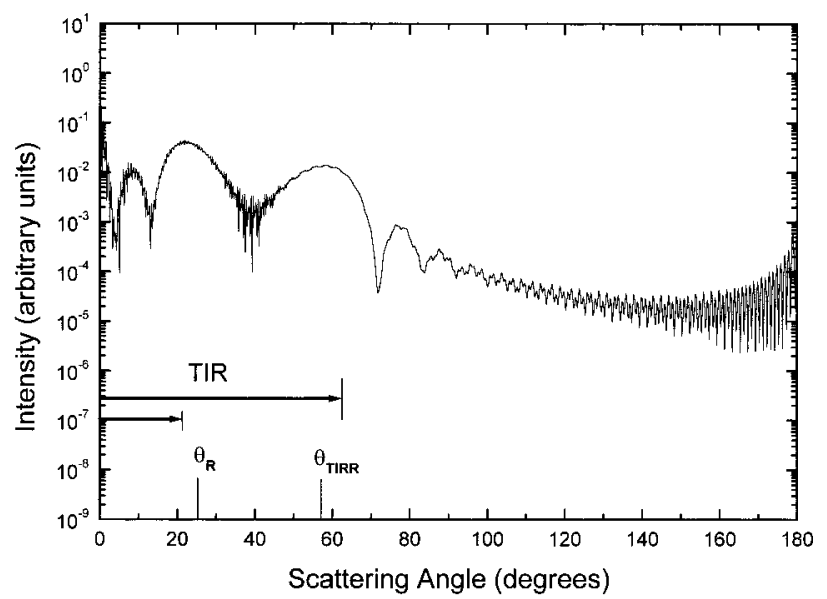

(d)

Fig. 10. Wave scattering intensity as a function of $\theta$ for $\phi=\pi / 2$ for an electric dipole source pointing in the $x$ direction with $\lambda=0.6328 \mu \mathrm{m}, a=30.0 \mu \mathrm{m}, N=4 / 3, q=0.99$, and (a) $p=1$, (b) $p=2$, (c) $p=3$, and (d) $p=4$. 


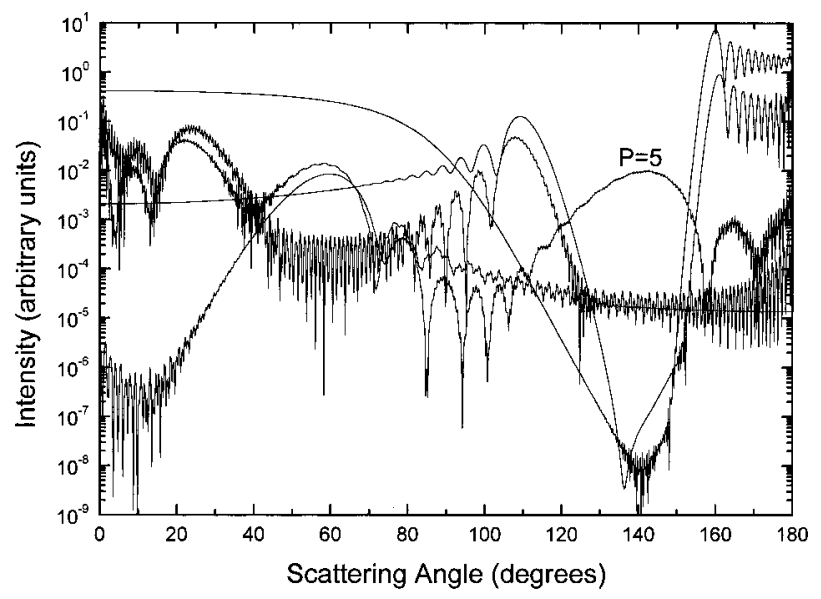

(a)

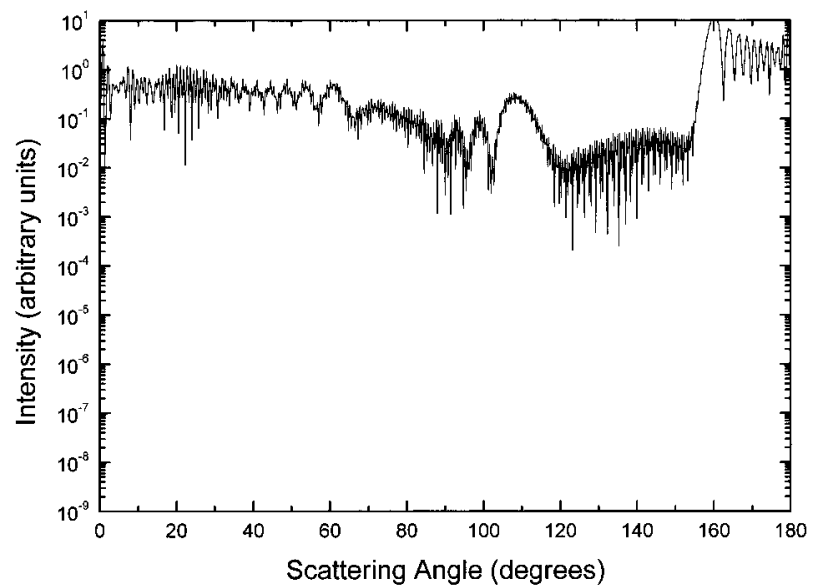

(b)

Fig. 11. (a) Wave scattering intensity of Figs. 10(a), 10(b), 10(c), and $10(\mathrm{~d})$ for $1 \leqslant p \leqslant 4$ along with the $p=5$ intensity, showing the confluence of the $p+1$ rainbow with the $p$ total internal reflection rainbow as $q \rightarrow 1$. (b) Total wave scattering intensity as a function of $\theta$ for $\phi=\pi / 2$ for an electric dipole source pointing in the $x$ direction with $\lambda=0.6328 \mu \mathrm{m}, a=30.0 \mu \mathrm{m}, N=4 / 3$, and $q=0.99$.

which coincide with the $p=2,3$ rainbows, are clearly visible in Fig. 11(b). The $p=4$ total internal reflection rainbow is beginning to emerge at $\theta \approx 60^{\circ}$ and is narrowed by its interference with $p=1$ radiation. The interference of the $p=5$ total internal reflection rainbow with the $p=6$ rainbow fills in the $120^{\circ} \leqq \theta \leqq 150^{\circ}$ total internal reflection region between the $p=1,2$ total internal reflection rainbows. The interference of the $p$ $=1,2,3,4$ wave families is also evident for $0^{\circ} \leqslant \theta$ $\lesssim 80^{\circ}$. The $p=3$ total internal reflection rainbow, along with the $p=4$ rainbow, is swallowed up by the $p$ $=1$ radiation and is not visible in the full scattered intensity.

The results presented thus far have been for scattering in the $y z$ plane of an $x$-direction radiating electric dipole inside a water droplet. To generalize these results, the wave scattering intensity for an orientation-averaged electric dipole source is shown in Figs. 12(a), 12(b), and 12 (c) for $\lambda=0.6328 \mu \mathrm{m}, a=30.0 \mu \mathrm{m}, N=4 / 3$, and $q$ $=0.10,0.748$, and 0.99, respectively. In Fig. 12(a) for $q=0.10$, the angle dependence of the scattered intensity is relatively featureless except for the oscillatory structure in the range $20^{\circ} \lesssim \theta \lesssim 160^{\circ}$. This is caused by interference of $p=1$ and 2 rays. The Fourier transform of this oscillatory structure within the aperture of an imaging lens produces the $p=1,2$ glare spots observed in the

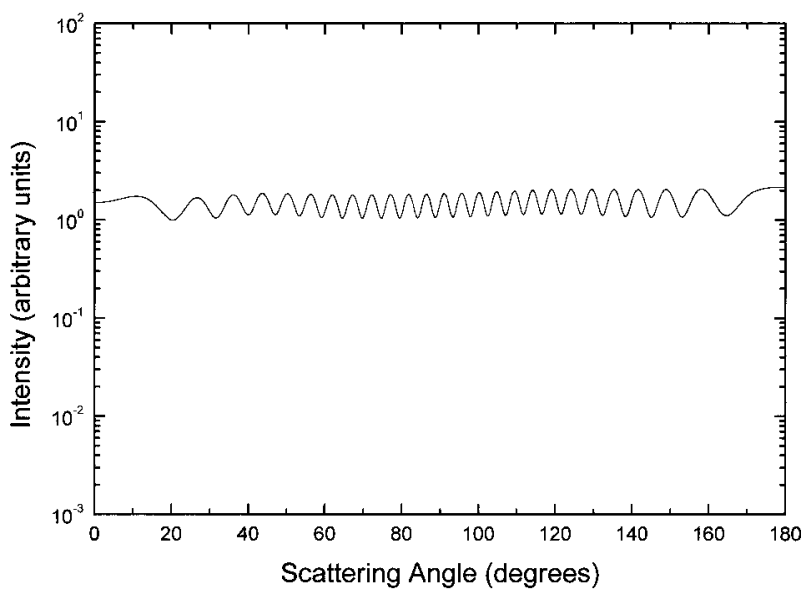

(a)

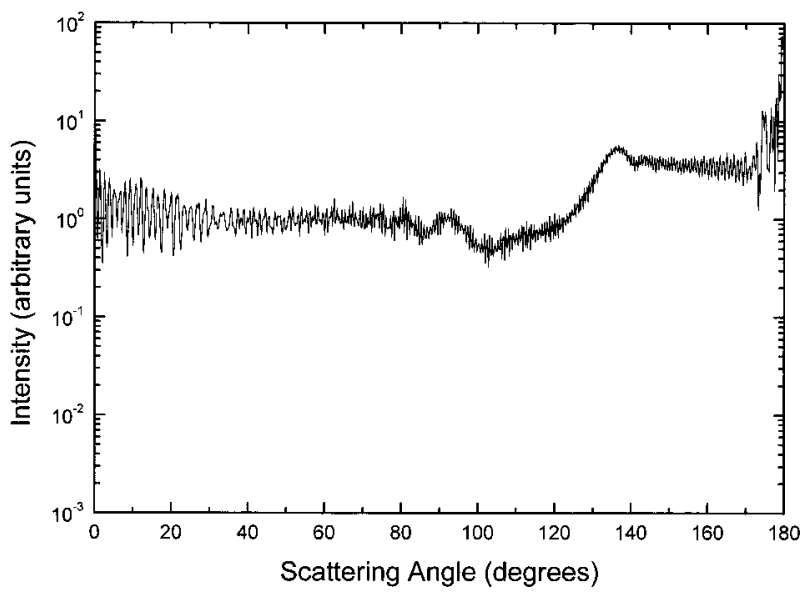

(b)

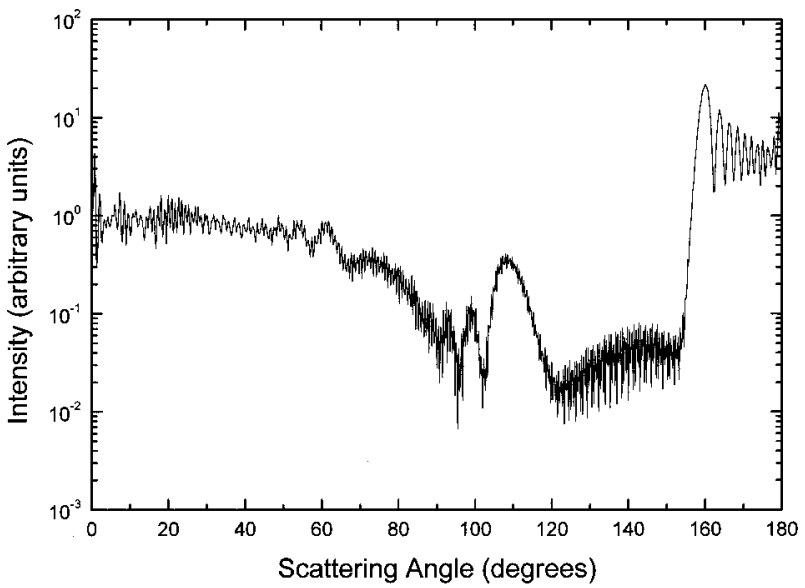

(c)

Fig. 12. Wave scattering intensity averaged over all electric dipole orientations as a function of $\theta$ for $\lambda=0.6328 \mu \mathrm{m}, a$ $=30.0 \mu \mathrm{m}, N=4 / 3$, and (a) $q=0.10$, (b) $q=0.75$, and (c) $q$ $=0.99$. 


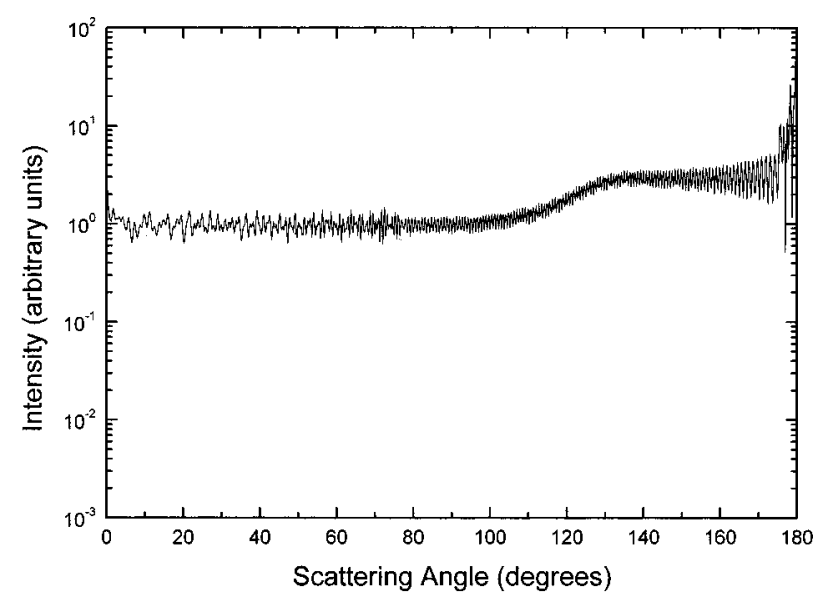

(a)

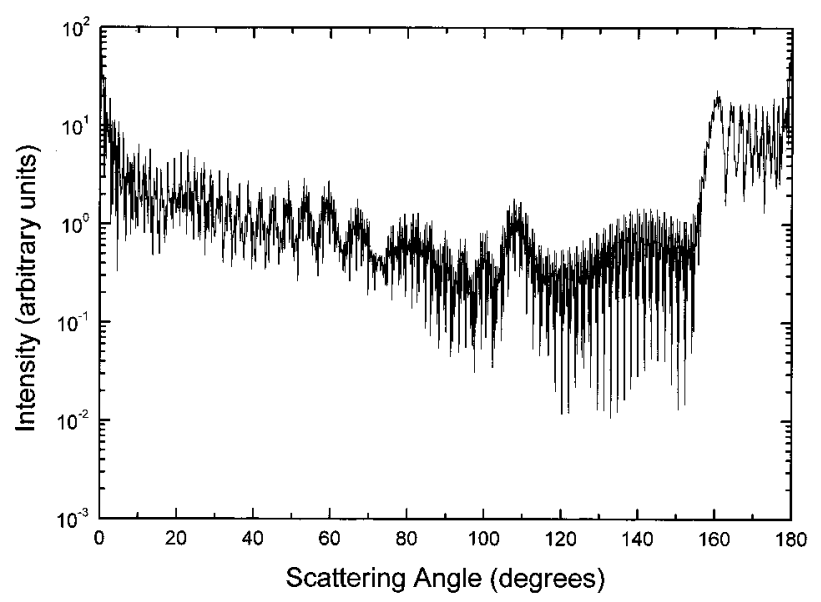

(b)

Fig. 13. Wave scattering intensity averaged over all electric dipole orientations as a function of $\theta$ for the conditions of the TEpolarized MDR in partial wave 309, $\lambda=0.6328 \mu \mathrm{m}, a$ $=29.940913 \mu \mathrm{m}, N=1.333333$, and (a) $q=0.70$ and (b) $q=0.99$.

image of the droplet in Ref. 35. As $q$ increases, the $p$ $=1,2$ interference structure in $I(\theta)$ becomes more rapid, implying that the $p=1,2$ glare spots in the image of the droplet grow farther apart. In Fig. 12(b) for $q$ $=0.748$, the $p=1$ resonance at $\theta \approx 130^{\circ}$, the $p=2$ rainbow at $\theta \approx 175^{\circ}$, the $p=2$ glory at $\theta \approx 180^{\circ}$, and the $p=5$ rainbow at $\theta \approx 95^{\circ}$ are clearly visible, and interference of the $p=1,2$, 3 rays dominates the intensity for $0^{\circ} \lesssim \theta \lesssim 80^{\circ}$. In Fig. 12(c) for $q=0.99$, the orientation-averaged-source scattered intensity is virtually identical to the intensity in the $\phi=\pi / 2$ plane for an $x$-direction electric dipole source in Fig. 11(b). This verifies the claim above that the scattered intensity of an isotropic point source is a good approximation to the orientation-averaged scattered intensity of an electric dipole source.

We have thus far tacitly assumed that the sphere size parameter does not correspond to an MDR, which occurs in the scattering amplitudes $c_{n}$ and $d_{n}$ for each partial wave in the interval $x \leqq n \lesssim N x$. For an interior source, MDR scattering is in general much stronger than Mie scattering of an incident plane wave by the sphere.
This is because the resonantly scattered intensity is proportional to the square of the resonant partial-wave contribution superimposed on a low-level background produced by all the other partial waves. For Mie scattering, the partial wave scattering amplitudes at resonance are constrained to $a_{n}=1$ and $b_{n}=1$ by energy conservation, while $\left|c_{n}\right|$ and $\left|d_{n}\right|$ for an interior source at resonance can become orders of magnitude larger.

As an example, an MDR with a full width at halfmaximum $\Delta a_{\mathrm{FWHM}}=6.56 \times 10^{-4} \mu \mathrm{m}$ occurs in the interior source partial wave scattering amplitude $d_{309}$ for $a=29.940913 \mu \mathrm{m}, \lambda=0.6328 \mu \mathrm{m}$, and $N=1.333333$. The orientation-averaged-source scattered intensity for these conditions is shown for $q=0.70$ and 0.99 in Figs. 13(a) and 13(b), respectively. A ray undergoing repeated total internal reflections comes no closer to the center of the sphere than $q=1 / N=0.75$. As a result, a source at $q=0.70$, as in Fig. 13(a), cannot access the MDR mode, and the scattered intensity strongly resembles that for a nonresonant sphere size parameter. But totally internally reflected rays for a source at $q=0.99$ can be trapped in an MDR mode. As a result, the scattered intensity of Fig. 13(b) exhibits a resonant oscillatory structure proportional to the angular function $\left[\tau_{309,1}(\theta)\right]^{2} \mathrm{su}-$ perimposed on the nonresonant intensity of Fig. 12(c).

\section{SEMICLASSICAL SCATTERING OF A DISTRIBUTION OF ELECTRIC DIPOLE SOURCES}

In Section 3, we considered scattering by a single fluorescing molecule inside a spherical droplet. We now inquire whether any of the semiclassical phenomena observed in that situation persist if the droplet contains a weak uniform concentration of fluorescing molecules and the entire droplet volume emits electric dipole radiation. Consider an unpolarized plane wave of wavelength $\lambda_{s}$ traveling in the positive $z$ direction and incident on the droplet. In quantum-mechanical perturbation theory, the probability that the field inside the droplet at the position $\left(r_{s}, \theta_{s}, \phi_{s}\right)$ produced by the plane wave excites a molecular transition to the fluorescent excited state is proportional to the interior source function $\left|\mathbf{E}_{\text {interior }}\left(\lambda_{s} ; r_{s}, \theta_{s}, \phi_{s}\right)\right|^{2}$. The total incoherent scattered intensity that is due to all the orientation-averaged sources inside the droplet is then

$$
\begin{aligned}
I_{\text {total }}^{\text {ave }}(r, \theta, \phi) \propto & \int \mathrm{d} \lambda_{f} \int \mathrm{d}^{3} r_{s}\left|E_{\text {interior }}\left(\lambda_{s} ; r_{s}, \theta_{s}, \phi_{s}\right)\right|^{2} \\
& \times S\left(\lambda_{f}\right) I_{\text {scatt }}^{\text {ave }}\left(\lambda_{f} ; r, \theta^{\prime}\right)
\end{aligned}
$$

where $S\left(\lambda_{f}\right)$ is the fluorescence intensity spectrum of the molecules and $I_{\text {scatt }}^{\text {ave }}\left(\lambda_{f} ; r, \theta^{\prime}\right)$ is the orientation-averaged intensity of Eq. (40) for a single source of unit strength at the fluorescent wavelength $\lambda_{f}$ on the positive $z$ axis at $z_{s}$, and the angle $\theta^{\prime}$ is given by ${ }^{36}$

$$
\cos \left(\theta^{\prime}\right)=\cos (\theta) \cos \left(\theta_{s}\right)+\sin (\theta) \sin \left(\theta_{s}\right) \cos \left(\phi-\phi_{s}\right) .
$$


If the imaginary part of the droplet's refractive index at $\lambda_{s}$ is small, the interior source function is dominated by the cusp and axial caustics of the various $p$ wave families, with most of the regions of large source function occurring on or near the positive $z$ axis near the critical source position. ${ }^{27}$ Thus it is of interest to inquire whether the $p=1$ scattering resonance/total internal reflection rainbow of Fig. $12(\mathrm{~b})$ at $\theta \approx 135^{\circ}$ remains visible in $I_{\text {total }}^{\text {ave }}(r, \theta, \phi) . \quad$ But in expression (48), the fluorescence intensity spectrum must be integrated over in order to include the effect of MDRs at angles where scattering is otherwise weak. In addition, all the weakly radiating sources throughout the droplet volume provides a large, nearly isotropic background intensity. As a result, the wave theory calculation shown in Fig. 2a of Ref. 37 indicates that the $p=1$ structure is not visible in the scattered intensity, and the experimental data shown in that figure confirm it. The strong backscattering enhancement predicted by reciprocity is the only identifiable feature seen experimentally. But this also is semiclassical in origin, being the incoherent sum of all the glories, the rainbow-enhanced glories, and the axial focusing described in Sections 2 and 3.

\section{ACKNOWLEDGMENTS}

The author thanks Michelle Zaller of the NASA-Glenn Research Center and Edward Hovenac of Federal Data Corp. and Valley Scientific Corp. for suggesting this line of research. This work was supported in part by the NASA-Glenn Research Center and Valley Scientific Corp. The author also thanks Steven Hill of the U.S. Army Research Laboratory for clarifying a number of issues on interior source scattering.

Address correspondence to the author at the location on the title page or by e-mail, jimandcarol@stratos.net.

\section{REFERENCES}

1. H. C. van de Hulst, Light Scattering by Small Particles (Dover, New York, 1981), pp. 119-126.

2. M. Kerker, The Scattering of Light and Other Electromagnetic Radiation (Academic, New York, 1969), pp. 39-49.

3. C. F. Bohren and D. R. Huffman, Absorption and Scattering of Light by Small Particles (Wiley, New York, 1983), pp. 82101.

4. P. Debye, "Das elektromagnetische feld um einen zylinder und die theorie des regenbogens," Phys. Z. 9, 775-778 (1908), reprinted and translated into English in P. L. Marston, ed., Selected Papers on Geometrical Aspects of Scattering (Society of Photo-Optical Instrumentation Engineers, Bellingham, Wash., 1994), pp. 198-204.

5. B. van der Pol and H. Bremmer, "The diffraction of electromagnetic waves from an electrical point source round a finitely conducting sphere, with applications to radiotelegraphy and the theory of the rainbow," Philos. Mag. 24, 141176, 825-864 (1937).

6. Ref. 1, pp. 210-214.

7. H. M. Nussenzveig, "High-frequency scattering by a transparent sphere. 1. Direct reflection and transmission," J. Math. Phys. 10, 82-124 (1969).

8. H. M. Nussenzveig, "High-frequency scattering by a transparent sphere. 2. Theory of the rainbow and the glory," J. Math. Phys. 10, 125-176 (1969).
9. H. M. Nussenzveig, Diffraction Effects in Semiclassical Scattering (Cambridge U. Press, Cambridge, UK, 1992), pp. $45-51$.

10. H. Chew, P. J. McNulty, and M. Kerker, "Model for Raman and fluorescent scattering by molecules embedded in small particles," Phys. Rev. A 13, 396-404 (1976).

11. H. Chew, "Transition rates of atoms near spherical surfaces," J. Chem. Phys. 87, 1355-1360 (1987).

12. S. D. Druger, S. Arnold, and L. M. Folan, "Theory of enhanced energy transfer between molecules embedded in spherical dielectric particles," J. Chem. Phys. 87, 26492659 (1987).

13. S. C. Hill, H. I. Saleheen, and K. A. Fuller, "Volume current method for modeling light scattering by inhomogeneously perturbed spheres," J. Opt. Soc. Am. A 12, 905-915 (1995).

14. S. C. Hill, H. I. Saleheen, M. D. Barnes, W. B. Whitten, and J. M. Ramsey, "Modeling fluorescence collection from single molecules in microspheres: effects of position, orientation, and frequency," Appl. Opt. 35, 6278-6288 (1996).

15. S. C. Hill, G. Videen, and J. D. Pendleton, "Reciprocity method for obtaining the far fields generated by a source inside or near a microparticle," J. Opt. Soc. Am. B 14, 25222529 (1997).

16. J. D. Pendleton and S. C. Hill, "Collection of emission from an oscillating dipole inside a sphere: analytical integration over a circular aperture," Appl. Opt. 36, 8729-8737 (1997).

17. M. Kerker and S. D. Druger, "Raman and fluorescent scattering by molecules embedded in spheres with radii up to several multiples of the wavelength," Appl. Opt. 18, 1172 1179 (1979).

18. J. Zhang and D. R. Alexander, "Hybrid inelastic-scattering models for particle thermometry: unpolarized emissions," Appl. Opt. 31, 7132-7139 (1992).

19. J. Zhang and D. R. Alexander, "Hybrid inelastic-scattering models for particle thermometry: polarized emissions," Appl. Opt. 31, 7140-7146 (1992).

20. J. Zhang and L. A. Melton, "Numerical simulations and restorations of laser droplet-slicing images," Appl. Opt. 33, 192-200 (1994).

21. N. Velesco and G. Schweiger, "Geometrical optics calculation of inelastic scattering on large particles,"Appl. Opt. 38, 1046-1052 (1999).

22. R. Domann and Y. Hardalupas, "Spatial distribution of fluorescence intensity within large droplets and its dependence on dye concentration," Appl. Opt. 40, 3586-3597 (2001).

23. P. L. Marston, "Geometrical and catastrophe optics methods in scattering," Phys. Acoust. 21, 1-234 (1992), p. 187, Fig. 76 .

24. D. S. Langley and M. J. Morrell, "Rainbow-enhanced forward and backward glory scattering," Appl. Opt. 30, 34593467 (1991).

25. C. F. Bohren and A. B. Fraser, "Newton's zero-order rainbow: unobservable or nonexistent?" Am. J. Phys. 59, 325326 (1991).

26. J. A. Lock and T. A. McCollum, "Further thoughts on Newton's zero-order rainbow," Am. J. Phys. 62, 1082-1089 (1994).

27. J. A. Lock and E. A. Hovenac, "Internal caustic structure of illuminated liquid droplets," J. Opt. Soc. Am. A 8, 15411552 (1991).

28. S. C. Hill, M. D. Barnes, W. B. Whitten, and J. M. Ramsey, "Collection of fluorescence from single molecules in microspheres: effects of illumination geometry," Appl. Opt. 36, 4425-4437 (1997).

29. E. A. Hovenac and J. A. Lock, "Assessing the contributions of surface waves and complex rays to far-field Mie scattering by use of the Debye series," J. Opt. Soc. Am. A 9, 781795 (1992).

30. H. M. Nussenzveig, "Complex angular momentum theory of the rainbow and the glory," J. Opt. Soc. Am. 69, 1068-1079, 1193-1194 (1979).

31. J. A. Lock and C. L. Adler, "Debye series analysis of the first order rainbow produced in scattering of a diagonally incident plane wave by a circular cylinder," J. Opt. Soc. Am. A 14, 1316-1328 (1997). 
32. C. M. Mount, D. B. Theissen, and P. L. Marston, "Scattering observations for tilted transparent fibers: evolution of the Airy caustics with cylinder tilt and the caustic merging transition," Appl. Opt. 37, 1534-1539 (1998).

33. D. Q. Chowdhury, D. H. Leach, and R. K. Chang, "Effect ofthe Goos-Hanchen shift on the geometrical-optics model for spherical-cavity mode spacing," J. Opt. Soc. Am. A 11, 11101116 (1994).

34. N. H. Tran, L. Dutriaux, Ph. Balcou, A. Le Floch, and F. Bretenaker, "Angular Goos-Hanchen effect in curved dielectric microstructures,” Opt. Lett. 20, 1233-1235 (1995).

35. S. C. Hill, P. Nachman, S. Arnold, J. M. Ramsey, and M. D. Barnes, "Fluorescence image of a single molecule in a microscope: model," J. Opt. Soc. Am. B 16, 1868-1873 (1999).

36. Ref. 35, footnote 30.

37. S. C. Hill, V. Boutou, J. Yu, S. Ramstein, J.-P. Wolf, Y.-L. Pan, S. Holler, and R. K. Chang, "Enhanced backwarddirected multiphoton-excited fluorescence from dielectric microcavities," Phys. Rev. Lett. 85, 54-57 (2000). 